\title{
Sex-related Differences in Patients with Positive Coronary Spasm as Identified by Acetylcholine Testing
}

\author{
Shozo Sueda ${ }^{1}$ and Tomoki Sakaue ${ }^{2}$
}

\begin{abstract}
:
Objective A pathological acetylcholine (ACh) test was observed at lower ACh doses in females compared with males in European populations. We retrospectively analyzed the sex-related differences in Japanese patients with provoked positive spasm by ACh spasm provocation testing.

Methods We performed the ACh spasm provocation tests in 1,854 patients from Jan 1991 until Mar 2019. ACh was injected in incremental doses of 20/50/100/200 $\mu$ g into the left coronary artery and 20/50/80 $\mu \mathrm{g}$ into the right coronary artery. Positive spasm was defined as $>90 \%$ stenosis and usual chest pain or ischemic ECG changes. We compared the clinical characteristics, angiographical findings during ACh testing, and clinical outcomes between female and male patients with and without provoked positive spasm.

Results Positive provoked spasm was diagnosed in 917 patients including 737 (80.4\%) males and 180 $(19.6 \%)$ females. The incidence of provoked positive spasm in females was significantly lower than that in males $(33.5 \%$ vs. $56.0 \%, \mathrm{p}<0.001)$. Female patients with provoked positive spasm tended to be older, have less history of smoking, less provoked spasm in the left circumflex artery, or less focal type spasm than male patients with provoked positive spasm. The incidence of ST elevation during ACh testing in male patients was significantly higher than that in female patients, whereas the frequency of ST depression in females was remarkably higher than that in males. The mean maximum used ACh dose for provoked positive spasm on both coronary arteries in female patients was significantly higher than that in male patients. The observed major complications during ACh testing did not differ substantially between the sexes. In addition, the prognosis in females with provoked positive spasm was not different from males.

Conclusion Provoked positive spasm by ACh test was obtained at lower mean maximum ACh doses in males compared with females in Japanese patients.
\end{abstract}

Key words: acetylcholine, male, female, sex-related difference, positive spasm

(Intern Med 60: 2357-2365, 2021)

(DOI: 10.2169/internalmedicine.6630-20)

\section{Introduction}

Intracoronary acetylcholine testing is clinically useful for the documentation of coronary artery spasm (1-3). We reported the super-sensitivity of acetylcholine (ACh) in comparison to ergonovine in Japanese female patients (4). Aziz et al. reported the super-sensitivity of ACh testing in European female patients with angina and unobstructed coronary arteries compared with those male patients, because a pathological ACh test was observed at lower ACh doses in fe- males compared with males (5). However, we had no data about sex-related differences in Japanese patients with positive provoked spasm by ACh testing.

In this article, we investigated the sex differences concerning the clinical and angiographical characteristics during ACh spasm provocation tests, and clinical outcomes in Japanese patients with and without positive provoked spasm. We also compared the sex-related differences to ACh between the Japanese investigated in our study and European populations evaluated by Aziz et al.

${ }^{1}$ Department of Cardiology, Ehime Prefectural Niihama Hospital, Japan and ${ }^{2}$ Department of Cardiology, Yawatahama City General Hospital, Japan

Received: November 13, 2020; Accepted: December 26, 2020; Advance Publication by J-STAGE: February 15, 2021

Correspondence to Dr. Shozo Sueda, EZF03146@nifty.com 


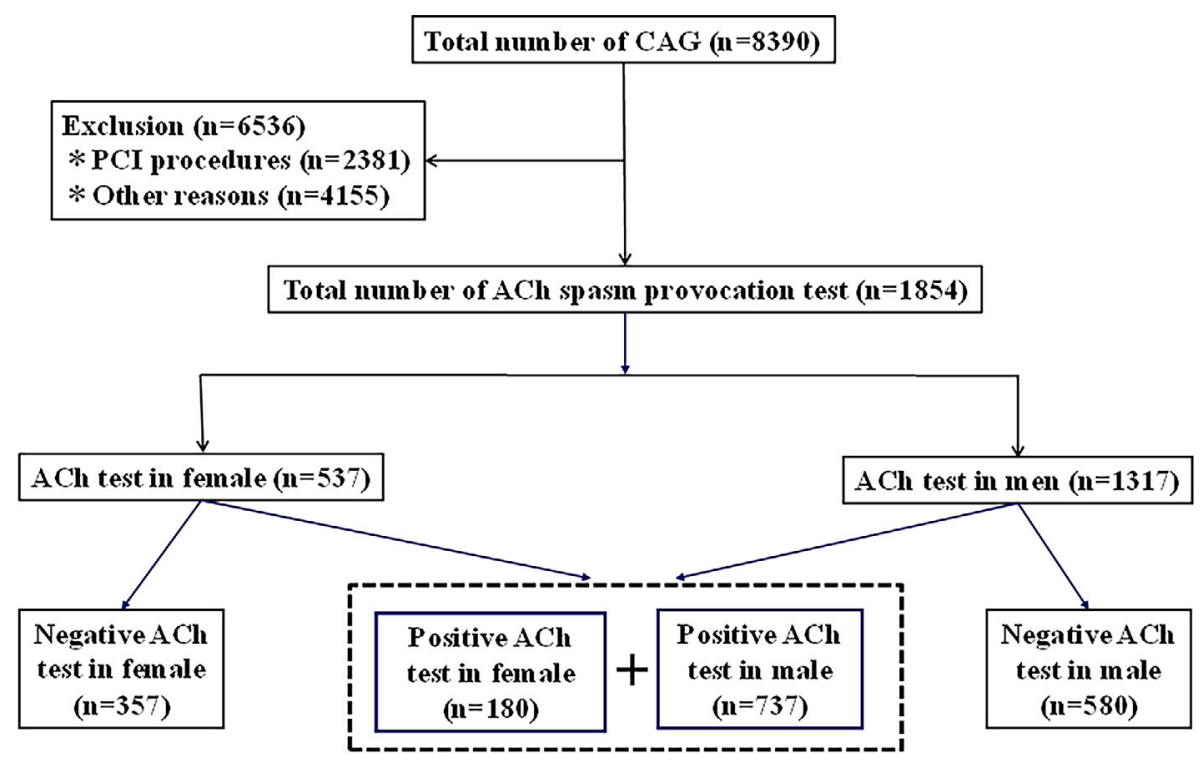

(ACh: acetylcholine, PCI: percutaneous coronary intervention)

Figure 1. Flow chart of the acetylcholine spasm provocation tests.

\section{Materials and Methods}

\section{Study patients}

This is a single-center, retrospective, and observational study. From January 1991 to March 2019, we performed a total of 8,390 coronary angiography procedures including 2,381 percutaneous coronary intervention procedures and 6,009 diagnostic and follow-up cardiac catheterization procedures, as shown in Fig. 1. During the same time, we performed 1,854 ACh spasm provocation tests and we tried to perform the selective spasm provocation tests to examine the incidence of provoked spasm in patients who had undergone coronary angiography whenever possible. To be more specific, we performed ACh spasm provocation tests in patients with and without obstructive coronary arteries and in patients with and without ischemic heart diseases, when coronary spasm was suspected by anamnesis and non-invasive tests. The subjects were excluded and the provocation test was not performed if patients had left main narrowing (> $50 \%$ ), triple-vessel disease, two-vessel disease with total occlusion, heart failure (New York Heart Association functional class III or IV), renal failure (creatinine $>2.0 \mathrm{mg} / \mathrm{dL}$ ), if spontaneous spasm was observed or if isosorbide dinitrate was initially used to relieve spasm in the coronary artery tested.

\section{The definition of usual chest pain, positive spasm} and spasm configuration type

We defined usual chest pain during ACh testing as the reproduction of the same chest symptoms complained. We also defined unusual chest pain during ACh tests as the different chest symptoms complained. We defined ACh positive spasm as $>90 \%$ transient stenosis and usual chest symptom or ischemic ECG changes. We also defined definite vasospastic angina as $>90 \%$ transient luminal narrowing with sings/symptoms of myocardial ischmeia (6). Focal spasm was defined as a discrete transient vessel narrowing of $>90 \%$ localized in the major coronary artery. Diffuse spasm was diagnosed when transient vessel narrowing was $>90 \%$ compared to the baseline coronary angiography observed from the proximal to the distal segment in the three major coronary arteries. We classified the positive provoked spasm patients into the 3 patterns consisting of having just focal type, just diffuse type, or combined type. The combined type was defined as having both focal and diffuse spasm. The degree of ST-segment depression was measured 80 msec after the $\mathrm{J}$ point. We considered a result to be positive when at least 1 of the following ischemic ECG changes was demonstrated during and/or after the ACh test: (1) STsegment elevation of $\geq 0.1 \mathrm{mV}$ in at least 2 contiguous leads; (2) ST-segment depression of $0.1 \mathrm{mV}$ in at least two contiguous leads. We also considered a negative $U$ wave to indicate a positive ischemic ECG change.

The definition of major complications during $\mathrm{ACh}$ testing and major cardiac events during follow-up periods

We defined major complications during ACh testing as shock $(<60 \mathrm{mmHg})$, ventricular fibrillation, sustained or non-sustained ventricular tachycardia, or fast paroxysmal atrial fibrillation. We also defined major cardiac events (MACEs) during follow-up periods as unstable angina necessary for admission, sudden cardiac death (SCD), ventricular fibrillation/tachycardia, heart failure, cerebral infraction, percutaneous coronary intervention, or acute coronary syndrome (ACS). 


\section{Spasm provocation test}

All drugs except for nitroglycerine were discontinued for $\geq 24$ hours before the study, and nitroglycerine was also discontinued $\geq 4$ hours before the study. Cardiac catheterization was performed from 9:00 am to 4:00 pm in the fasting state. After control coronary arteriograms of the left coronary artery (LCA) in the right anterior oblique with caudal projection and of the right coronary artery (RCA) in the left anterior oblique with cranial projection were obtained by injection of 8-10 mL of contrast medium, a temporary pacemaker was inserted into the right ventricle of each ACh testing patient and the pacing rate was set at $40-45$ beats/min.

Provocation of coronary artery spasm was performed with an intracoronary injection of $\mathrm{ACh}$, as previously reported (7). ACh chloride (Neucholin-A, $30 \mathrm{mg} / 2 \mathrm{~mL}$; Zeria Seiyaku, Tokyo, Japan) was injected in incremental doses of 20, 50 and $80 \mu \mathrm{g}$ into the RCA and of 20, 50, 100 and 200 $\mu \mathrm{g}$ into the LCA over 20 seconds with at least a 3-minute interval between each injection. Coronary arteriography was performed when ST-segment changes and/or, chest pain occurred or 1-2 minutes after the completion of each injection. When an induced coronary spasm did not resolve spontaneously within 3 minutes after the completion of ACh injections or when hemodynamic instability occurred as the result of coronary spasm, 2.5 to $5.0 \mathrm{mg}$ of nitrate was injected into the involved vessel. A standard 12-lead electrocardiogram was recorded every 30 seconds. We used the ECG findings when $\mathrm{ACh}$, saline and contrast medium were not injected into the responsible vessels for at least 60 seconds. After the spasm provocation tests were completed, an intracoronary injection of $5.0 \mathrm{mg}$ isosorbide dinitrate was administered, and coronary arteriography was then performed in multiple projections.

During the study, the arterial blood pressure and ECG were continuously monitored on an oscilloscope by NihonKohden polygraphy (Tokyo, Japan). In the present study, coronary arteriograms were analyzed separately by 2 independent observers. The percent luminal diameter narrowing of the coronary arteries was measured using an automatic edge-counter detection computer analysis system. The size of the coronary catheter was used to calibrate the images in millimeters and the measurement was performed in the same projection of coronary angiography at each stage. Any patients with catheter-induced spasms were excluded from this study. Significant organic stenosis was defined as $>75 \%$ luminal narrowing according to the American College of Cardiology/American Heart Association classification (8).

The study protocol complied with the Declaration of Helsinki. Written informed consent was obtained from all patients before performing the pharmacological spasm provocation tests and the protocol of this study was in agreement with the guidelines of the ethical committee at our institutions.

\section{Statistical analysis}

A data analysis was carried out using the SPSS software program (version 22.0, IBM Japan, Tokyo, Japan). All data were presented as the mean \pm SD. The clinical characteristic including coronary risk factors, provoked positive spasm incidence, occurrence of major complications, or medications were analyzed by the Fisher's exact test with correction or the Mann-Whitney U test. Mean maximum used ACh dose for provoked positive spasms was also analyzed by the Mann-Whitney $U$ test. Event-free survival curves from MACEs were constructed using the Kaplan-Meier survival method. $\mathrm{P}<0.05$ was considered significant.

\section{Results}

\section{Comparisons of all patient clinical characteristics}

Table 1 showed the clinical characteristics in 1,854 patients with ACh testing. The prevalence of ischemic heart disease, a history of smoking, the administration of calciumchannel-blocker (CCB)s/aspirin/nitrates were frequently observed in males, while higher age and higher ejection fraction of left ventricle by ultrasonography was often recognized in females.

\section{Comparisons of clinical background in patients who were ACh positive}

As shown in Table 2, we provoked positive spasm in 917 patients (49.5\%) including 180 female patients and 737 male patients. Ischemic heart disease was observed in 802 patients, while non-ischemic heart disease was recognized in 115 patients. Obstructive coronary artery disease (>75\%) was recognized in 201 patients $(21.9 \%)$, while 724 patients (79.0\%) had a history of smoking. Hypertension was observed in 418 patients $(45.6 \%)$, whereas 479 patients (52.2\%) had dyslipidemia. Diabetes mellitus was found in 244 patients $(26.6 \%)$. We could perform spasm provocation tests on both coronary arteries in 843 (91.9\%) patients with provoked positive spasm. We could perform spasm provocation tests of either one vessel in 61 male patients including 44 RCAs and 17 LCAs, while we also could perform these testing of either one vessel in 13 female patients including 11 RCAs and 2 LCAs. Female patients with provoked positive spasm tended to be older, have less history of smoking, a higher ejection fraction, a higher level of all serum lipid examinations except for triglyceride compared with those for male patients.

\section{Comparisons of the angiographical characteristics between male and female patients}

The incidence of provoked positive spasm in females was significantly lower than that in males $(33.5 \%$ vs. $56.0 \%, \mathrm{p}<$ 0.001), as shown in Table 3. Furthermore, females had less positive provoked spasm in the left circumflex artery or less focal and combined type spasm compared with males. 
Table 1. All Patient Clinical Characteristics.

\begin{tabular}{lcccc}
\hline & Total & Female & Male & p value \\
\hline Number & 1,854 & 537 & 1,317 & \\
IHD & $1,320(71.2 \%)$ & $328(61.1 \%)$ & $992(75.3 \%)$ & $<0.001$ \\
Non-IHD & $534(28.8 \%)$ & $209(38.9 \%)$ & $325(24.7 \%)$ & $<0.001$ \\
Follow up duration (month) & $47.8 \pm 30.6$ & $47.9 \pm 30.6$ & $47.8 \pm 30.8$ & 0.9061 \\
Age (year) & $64.8 \pm 10.7$ & $67.6 \pm 9.2$ & $63.8 \pm 11.0$ & $<0.001$ \\
Obstructive CAD (>75\%) & $405(21.8 \%)$ & $101(18.8 \%)$ & $304(23.1 \%)$ & 0.0433 \\
Hypertension & $830(44.8 \%)$ & $243(45.3 \%)$ & $587(44.6 \%)$ & 0.7892 \\
Dyslipidemia & $840(45.3 \%)$ & $230(42.8 \%)$ & $560(42.5 \%)$ & 0.9026 \\
Diabetes mellitus & $465(25.1 \%)$ & $120(22.3 \%)$ & $345(26.2 \%)$ & 0.0828 \\
Smoking & $1,251(64.5 \%)$ & $41(7.6 \%)$ & $1,210(91.9 \%)$ & $<0.001$ \\
EF by UCG (\%) & $63 \pm 11$ & $65 \pm 10$ & $62 \pm 12$ & $<0.001$ \\
Total cholesterol (mg/dL) & $191.0 \pm 36.1$ & $197.9 \pm 34.1$ & $188.4 \pm 36.6$ & $<0.001$ \\
Triglyceride (mg/dL) & $134.2 \pm 84.9$ & $113.0 \pm 60.0$ & $141.5 \pm 90.5$ & $<0.001$ \\
LDL-cholesterol (mg/dL) & $114.7 \pm 31.5$ & $120.0 \pm 30.5$ & $112.9 \pm 31.9$ & $<0.001$ \\
HDL-cholesterol (mg/dL) & $48.9 \pm 13.1$ & $52.7 \pm 12.9$ & $47.3 \pm 12.7$ & $<0.001$ \\
Fast blood glucose (mg/dL) & $114.7 \pm 31.5$ & $108.6 \pm 35.8$ & $113.5 \pm 39.1$ & $<0.001$ \\
Glycohemoglobin (\% JDS) & $5.7 \pm 1.0$ & $5.7 \pm 0.9$ & $5.7 \pm 1.0$ & 0.7965 \\
Calcium channel blocker & $931(50.2 \%)$ & $214(39.9 \%)$ & $717(54.4 \%)$ & $<0.001$ \\
$\quad$ Dihydropyridine & $646(34.8 \%)$ & $154(28.7 \%)$ & $492(37.4 \%)$ & $<0.001$ \\
Benzodiazepine & $379(20.4 \%)$ & $75(14.0 \%)$ & $304(23.1 \%)$ & $<0.001$ \\
ARB or ACEI & $351(18.9 \%)$ & $106(19.7 \%)$ & $245(18.6 \%)$ & 0.5710 \\
Beta blocker & $198(10.7 \%)$ & $66(12.3 \%)$ & $132(10.0 \%)$ & 0.1515 \\
Aspirin & $585(31.6 \%)$ & $120(22.3 \%)$ & $465(35.3 \%)$ & $<0.001$ \\
Anti-platelet & $301(16.2 \%)$ & $71(13.2 \%)$ & $230(17.5 \%)$ & 0.0246 \\
Nitrate & $520(28.0 \%)$ & $84(15.6 \%)$ & $436(33.1 \%)$ & $<0.001$ \\
Nitrate tape & $208(11.2 \%)$ & $55(10.2 \%)$ & $153(11.6 \%)$ & 0.3947 \\
Nicorandil & $208(11.2 \%)$ & $65(12.1 \%)$ & $143(10.9 \%)$ & 0.4405 \\
Statin & $417(22.5 \%)$ & $108(20.1 \%)$ & $309(23.5 \%)$ & 0.1170 \\
\hline
\end{tabular}

IHD: ischemic heart disease, CAD: coronary artery disease, EF: ejection fraction, UCG: ultrasound cardiography, LDL: low-density-lipoprotein, HDL: high-density-lipoprotein, JDS: Japan Diabetes Society, ARB: angiotensin receptor blocker, ACEI: angiotensin converting enzyme inhibitor

\section{Comparisons of chest symptoms and ischemic ECG changes during ACh testing}

As shown in Table 4, in the ACh testing of RCA, the incidence of ST elevation in female patients was significantly lower than that in male patients $(23.4 \%$ vs. $44.7 \%$, p < 0.001 ), while the frequency of ST depression in females was markedly higher than that in males $(45.3 \%$ vs. $33.1 \%$, p < $0.01)$. There was no difference regarding the incidence of usual chest pain complained during ACh tests between the sexes. In contrast, in the ACh tests of LCA, we also found at higher incidence of ST elevation $(30.3 \%$ vs. $18.2 \%$, p $<$ $0.01)$ and at lower frequency of ST depression $(35.7 \%$ vs. $50.4 \%, \mathrm{p}<0.01)$ in male patients compared with female those as well as RCA testing. The frequency of complaints of usual or unusual chest pain was not different between the sexes, while no complaints of chest pain in males was significantly higher than that in females $(14.9 \%$ vs. $8.0 \%$, p < 0.05). We also analyzed the same issues in 716 patients without obstructive coronary arteries. We could find closely similar results between all $917 \mathrm{ACh}$ positive patients and those with nonobstructive coronary arteries. Chest symptoms and ischemic ECG changes during ACh tests did not differ substantially between male and female patients with obstructive coronary arteries. There were no differences regarding chest symptoms and ischemic ECG changes during ACh testing between female patients with and without obstructive coronary arteries on both RCA and LCA, while the incidence of ischemic ECG changes [78.0\% (110/141) vs. $62.3 \%$ (288/462), $\mathrm{p}<0.001]$ and usual chest pain $(85.8 \%$ vs. $76.4 \%, \mathrm{p}=0.0170$ ) on LCA was significantly higher in male patients with obstructive coronary arteries than in those without them.

\section{Comparisons of maximum ACh dose used for posi- tive provoked spasm}

In the RCA, the incidence of the maximum ACh dose used for provoked positive spasm was not different between male and female patients as shown in Table 5. However, the mean used maximum ACh dose for provoked positive spasm in females was markedly higher than that in males $(59 \pm 21$ $\mu \mathrm{g}$ vs. $54 \pm 23 \mu \mathrm{g}, \mathrm{p}=0.0417)$. In the LCA, we also found no differences in the frequency of maximum ACh dose used for positive provoked spasm between male and female patients. However, the mean used maximum ACh dose for provoked positive spasm in females was significantly higher than that 
Table 2. Clinical Background Data in All Study Patients.

\begin{tabular}{|c|c|c|c|c|c|c|}
\hline & Female positive & Male positive & $\mathrm{p}$ value & Female negative & Male negative & $\mathrm{p}$ value \\
\hline Number & 180 & 737 & & 357 & 580 & \\
\hline IHD & $157(87.2 \%)$ & $645(87.5 \%)$ & 0.9148 & $171(47.9 \%)$ & $347(59.8 \%)$ & $<0.001$ \\
\hline Non-IHD & $23(12.8 \%)$ & $92(12.5 \%)$ & 0.9148 & $186(52.1 \%)$ & $233(40.2 \%)$ & $<0.001$ \\
\hline Follow up duration (m) & $51.1 \pm 30.3$ & $48.6 \pm 30.9$ & 0.3197 & $46.3 \pm 30.7$ & $46.7 \pm 30.1$ & 0.8730 \\
\hline Age (year) & $68.1 \pm 9.5$ & $64.4 \pm 10.4$ & $<0.001$ & $67.2 \pm 9.1$ & $63.0 \pm 11.8$ & $<0.001$ \\
\hline Obstructive CAD (>75\%) & $31(17.2 \%)$ & $170(23.1 \%)$ & 0.0893 & $70(19.6 \%)$ & $134(23.1 \%)$ & 0.2079 \\
\hline Hypertension & $87(48.3 \%)$ & $331(44.9 \%)$ & 0.4086 & $156(43.7 \%)$ & $256(44.1 \%)$ & 0.8950 \\
\hline Dyslipidemia & $95(52.8 \%)$ & $384(52.1 \%)$ & 0.8709 & $135(37.8 \%)$ & $226(39.0 \%)$ & 0.7252 \\
\hline Diabetes mellitus & $43(23.9 \%)$ & $201(27.3 \%)$ & 0.3570 & $77(21.6 \%)$ & $144(24.8 \%)$ & 0.2538 \\
\hline Smoking & $28(15.6 \%)$ & $696(94.4 \%)$ & $<0.001$ & $13(3.6 \%)$ & $514(88.6 \%)$ & $<0.001$ \\
\hline EF by UCG $(\%)$ & $68 \pm 10$ & $62 \pm 11$ & $<0.001$ & $64 \pm 10$ & $61 \pm 12$ & $<0.001$ \\
\hline Total cholesterol (mg/dL) & $200.8 \pm 35.5$ & $189.1 \pm 36.9$ & $<0.001$ & $195.7 \pm 32.8$ & $187.6 \pm 35.8$ & 0.0076 \\
\hline Triglyceride (mg/dL) & $100.7 \pm 44.4$ & $145.8 \pm 93.3$ & $<0.001$ & $123.4 \pm 69.0$ & $134.6 \pm 86.5$ & 0.1047 \\
\hline LDL-cholesterol (mg/dL) & $120.6 \pm 31.7$ & $112.8 \pm 32.2$ & $<0.01$ & $119.1 \pm 29.5$ & $113.0 \pm 31.0$ & 0.0326 \\
\hline HDL-cholesterol (mg/dL) & $55.1 \pm 12.7$ & $47.5 \pm 12.8$ & $<0.001$ & $51.0 \pm 13.0$ & $47.4 \pm 12.8$ & 0.0027 \\
\hline Fast blood glucose $(\mathrm{mg} / \mathrm{dL})$ & $111.1 \pm 40.6$ & $113.7 \pm 38.6$ & 0.4269 & $106.3 \pm 30.8$ & $113.0 \pm 39.5$ & 0.0371 \\
\hline Glycohemoglobin (\% JDS) & $5.7 \pm 0.9$ & $5.7 \pm 1.0$ & 0.6367 & $5.7 \pm 1.0$ & $5.7 \pm 1.0$ & 0.6277 \\
\hline Calcium channel blocker & $107(59.4 \%)$ & $461(62.6 \%)$ & 0.4416 & $147(41.2 \%)$ & $296(51.0 \%)$ & 0.0041 \\
\hline Dihydropyridine & $87(48.3 \%)$ & $326(44.2 \%)$ & 0.3216 & $87(24.4 \%)$ & $186(32.1 \%)$ & 0.0144 \\
\hline Benzodiazepine & $31(17.2 \%)$ & $197(26.7 \%)$ & 0.0081 & $64(17.9 \%)$ & $127(21.9 \%)$ & 0.1429 \\
\hline $\mathrm{ARB}$ or ACEI & $41(22.8 \%)$ & $124(16.8 \%)$ & 0.0623 & $65(18.2 \%)$ & $121(20.9 \%)$ & 0.3224 \\
\hline Beta blocker & $18(10.0 \%)$ & $67(9.1 \%)$ & 0.7061 & $48(13.4 \%)$ & $65(11.2 \%)$ & 0.3068 \\
\hline Aspirin & $47(26.1 \%)$ & $298(40.4 \%)$ & 0.0004 & $73(20.4 \%)$ & $167(28.8 \%)$ & 0.0044 \\
\hline Anti-platelet & $34(18.9 \%)$ & $161(21.8 \%)$ & 0.3848 & $37(10.4 \%)$ & $69(11.9 \%)$ & 0.4720 \\
\hline Nitrate & $38(21.1 \%)$ & $296(40.2 \%)$ & $<0.001$ & $46(12.9 \%)$ & $140(24.1 \%)$ & $<0.001$ \\
\hline Nitrate tape & $34(18.9 \%)$ & $116(15.7 \%)$ & 0.3058 & $21(5.9 \%)$ & $37(6.4 \%)$ & 0.7591 \\
\hline Nicorandil & $28(15.6 \%)$ & $91(12.3 \%)$ & 0.2509 & $37(10.4 \%)$ & $52(9.0 \%)$ & 0.4782 \\
\hline Statin & $52(28.9 \%)$ & $201(27.3 \%)$ & 0.6637 & $56(15.7 \%)$ & $108(18.6 \%)$ & 0.2509 \\
\hline
\end{tabular}

IHD: ischemic heart disease, CAD: coronary artery disease, EF: ejection fraction, UCG: ultrasound cardiography, LDL: low-density-lipoprotein, HDL: high-density-lipoprotein, JDS: Japan Diabetes Society, ARB: angiotensin receptor blocker, ACEI: angiotensin converting enzyme inhibitor

Table 3. Comparisons of Positive Spasm by Acetylcholine Spasm Provocation Testing.

\begin{tabular}{lcccc}
\hline & Total & Female & Male & p value \\
\hline Total number of patients & 1,854 & 537 & 1,317 & \\
Provoked positive spasm & $917(49.5 \%)$ & $180(33.5 \%)$ & $737(56.0 \%)$ & $<0.001$ \\
Right coronary artery & $664(72.4 \%)$ & $126(70.0 \%)$ & $538(73.0 \%)$ & 0.4197 \\
Left circumflex artery & $298(32.5 \%)$ & $47(26.1 \%)$ & $251(34.1 \%)$ & 0.0413 \\
Left anterior descending artery & $679(74.0 \%)$ & $131(72.8 \%)$ & $548(74.4 \%)$ & 0.6651 \\
1 vessel spasm & $388(42.3 \%)$ & $86(47.8 \%)$ & $302(41.0 \%)$ & 0.0978 \\
2 vessel spasm & $334(36.4 \%)$ & $64(35.6 \%)$ & $270(36.6 \%)$ & 0.7873 \\
3 vessel spasm & $195(21.3 \%)$ & $30(16.7 \%)$ & $165(22.4 \%)$ & 0.0926 \\
Focal type spasm & $277(30.2 \%)$ & $37(20.6 \%)$ & $240(32.6 \%)$ & 0.0017 \\
Diffuse type spasm & $483(52.7 \%)$ & $125(69.4 \%)$ & $358(48.6 \%)$ & $<0.001$ \\
Combined type spasm & $157(17.1 \%)$ & $18(10.0 \%)$ & $139(18.9 \%)$ & 0.0046 \\
\hline
\end{tabular}

EF: ejection fraction, UCG: ultrasound cardiography, LDL: low-density-lipoprotein, HDL: highdensity-lipoprotein

in males $(104 \pm 52 \mu \mathrm{g}$ vs. $91 \pm 50 \mu \mathrm{g}, \mathrm{p}=0.0121)$. We could find no differences regarding the incidence of the maximum ACh dose used for positive provoked spasm between all 917 patients and those 716 patients without obstructive coronary arteries. However, the mean maximum used ACh dose for provoked positive spasm in the RCA in females without obstructive coronary arteries was higher than that in males, but not significantly so $(60 \pm 21 \mu \mathrm{g}$ vs. $55 \pm 23 \mu \mathrm{g}, \mathrm{p}=0.0885)$. In contrast, the mean maximum used ACh dose for provoked positive spasm in the LCA in female patients with nonob- 
Table 4. Comparisons of Chest Symptoms and ECG Changes during Acetylcholine Testing in Patients with Positive Spasm.

\begin{tabular}{|c|c|c|c|c|c|c|c|c|c|}
\hline & \multicolumn{3}{|c|}{ All patients } & \multicolumn{3}{|c|}{$\begin{array}{c}\text { Without obstructive coronary } \\
\text { arteries }\end{array}$} & \multicolumn{3}{|c|}{ With obstructive coronary arteries } \\
\hline & Female & Male & $\mathrm{p}$ value & Female & Male & $\mathrm{p}$ value & Female & Male & $\mathrm{p}$ value \\
\hline \multicolumn{10}{|l|}{ RCA } \\
\hline Number & 128 & 553 & & 104 & 434 & & 24 & 119 & \\
\hline ST elevation & $30(23.4 \%)$ & $247(44.7 \%)$ & $<0.001$ & $22(21.2 \%)$ & $187(43.1 \%)$ & $<0.001$ & $8(33.3 \%)$ & $60(50.4 \%)$ & 0.1262 \\
\hline ST depression & $58(45.3 \%)$ & $183(33.1 \%)$ & 0.0093 & $46(44.2 \%)$ & $145(33.4 \%)$ & 0.0383 & $12(50.0 \%)$ & $38(31.9 \%)$ & 0.0904 \\
\hline Non-ST change & $38(29.7 \%)$ & $119(21.5 \%)$ & 0.0480 & $34(32.7 \%)$ & $98(22.6 \%)$ & 0.0314 & $4(16.7 \%)$ & $21(17.6 \%)$ & 0.9081 \\
\hline Unknown & $2(1.6 \%)$ & $4(0.7 \%)$ & 0.6960 & $2(1.9 \%)$ & $4(0.9 \%)$ & 0.7236 & 0 & 0 & \\
\hline Usual chest pain & $95(74.2 \%)$ & $435(78.7 \%)$ & 0.2755 & $76(73.1 \%)$ & $335(77.7 \%)$ & 0.3751 & $19(79.2 \%)$ & $100(84.0 \%)$ & 0.5605 \\
\hline Unusual chest pain & $13(10.2 \%)$ & $36(6.5 \%)$ & 0.1503 & $11(10.6 \%)$ & $32(7.4 \%)$ & 0.2792 & $2(8.3 \%)$ & $4(3.4 \%)$ & 0.5821 \\
\hline No chest pain & $20(15.6 \%)$ & $82(14.8 \%)$ & 0.8199 & $17(16.3 \%)$ & $67(15.4 \%)$ & 0.8187 & $3(12.5 \%)$ & $15(12.6 \%)$ & 0.7465 \\
\hline \multicolumn{10}{|l|}{ LCA } \\
\hline Number & 137 & 603 & & 117 & 462 & & 20 & 141 & \\
\hline ST elevation & $25(18.2 \%)$ & $183(30.3 \%)$ & 0.0045 & $22(18.8 \%)$ & $133(28.8 \%)$ & 0.0293 & $3(15.0 \%)$ & $50(35.5 \%)$ & 0.1168 \\
\hline ST depression & $69(50.4 \%)$ & $215(35.7 \%)$ & 0.0014 & $57(48.7 \%)$ & $155(33.5 \%)$ & 0.0023 & $12(60.0 \%)$ & $60(42.6 \%)$ & 0.1419 \\
\hline Non-ST change & $43(31.4 \%)$ & $202(33.5 \%)$ & 0.6353 & $38(32.5 \%)$ & $172(37.2 \%)$ & 0.3397 & $5(25.0 \%)$ & $30(21.2 \%)$ & 0.7055 \\
\hline Unknown & 0 & $3(0.5 \%)$ & 0.9342 & 0 & $2(0.4 \%)$ & 0.8657 & 0 & $1(0.7 \%)$ & 0.2531 \\
\hline Usual chest pain & $112(81.8 \%)$ & $474(78.6 \%)$ & 0.4131 & $94(80.3 \%)$ & $353(76.4 \%)$ & 0.3648 & $18(90.0 \%)$ & $121(85.8 \%)$ & 0.8721 \\
\hline Unusual chest pain & $14(10.2 \%)$ & $39(6.5 \%)$ & 0.1243 & $12(10.3 \%)$ & $36(7.8 \%)$ & 0.3879 & $2(10.0 \%)$ & $3(2.1 \%)$ & 0.2260 \\
\hline No chest pain & $11(8.0 \%)$ & $90(14.9 \%)$ & 0.0338 & $11(9.4 \%)$ & $73(15.8 \%)$ & 0.0792 & 0 & $17(12.1 \%)$ & 0.2101 \\
\hline
\end{tabular}

RCA: right coronary artery, LCA: left coronary artery

Table 5. Comparisons of Used Maximum Acetylcholine Dose for Positive Provoked Spasm.

\begin{tabular}{|c|c|c|c|c|c|c|c|c|c|}
\hline & \multicolumn{3}{|c|}{ All patients } & \multicolumn{3}{|c|}{ Without obstructive coronary arteries } & \multicolumn{3}{|c|}{ With obstructive coronary arteries } \\
\hline & Female & Male & $\mathrm{p}$ value & Female & Male & $\mathrm{p}$ value & Female & Male & $\mathrm{p}$ value \\
\hline \multicolumn{10}{|l|}{ RCA } \\
\hline Number & 128 & 553 & & 104 & 434 & & 24 & 119 & \\
\hline $10 \mu \mathrm{g}$ & 0 & $13(2.4 \%)$ & 0.1636 & 0 & $11(2.5 \%)$ & 0.2096 & 0 & $2(1.7 \%)$ & 0.7541 \\
\hline $20 \mu \mathrm{g}$ & $18(14.1 \%)$ & $114(20.6 \%)$ & 0.0910 & $13(12.5 \%)$ & $83(19.1 \%)$ & 0.1130 & $5(20.8 \%)$ & $31(26.1 \%)$ & 0.5911 \\
\hline $50 \mu \mathrm{g}$ & $54(42.2 \%)$ & $219(39.6 \%)$ & 0.5907 & $44(42.3 \%)$ & $167(38.5 \%)$ & 0.4726 & $10(41.7 \%)$ & $52(43.7 \%)$ & 0.8546 \\
\hline $80 \mu \mathrm{g}$ & $56(43.8 \%)$ & $207(37.4 \%)$ & 0.1859 & $47(45.2 \%)$ & $173(39.9 \%)$ & 0.3206 & $9(37.5 \%)$ & $34(28.6 \%)$ & 0.3842 \\
\hline $\begin{array}{l}\text { Mean maximum } \\
\text { used dose }(\mu \mathrm{g})\end{array}$ & $59 \pm 21$ & $54 \pm 23$ & 0.0417 & $60 \pm 21$ & $55 \pm 23$ & 0.0885 & $55 \pm 23$ & $50 \pm 23$ & 0.3432 \\
\hline \multicolumn{10}{|l|}{ LCA } \\
\hline Number & 137 & 603 & & 117 & 462 & & 20 & 141 & \\
\hline $10 \mu \mathrm{g}$ & $1(0.7 \%)$ & $4(0.7 \%)$ & 0.6229 & $1(0.8 \%)$ & $2(0.4 \%)$ & 0.8783 & 0 & $2(1.4 \%)$ & 0.5873 \\
\hline $20 \mu \mathrm{g}$ & $9(6.6 \%)$ & $63(10.4 \%)$ & 0.1668 & $4(3.4 \%)$ & $43(9.3 \%)$ & 0.0582 & $5(25.0 \%)$ & $20(14.2 \%)$ & 0.2113 \\
\hline $50 \mu \mathrm{g}$ & $24(17.5 \%)$ & $140(23.2 \%)$ & 0.1471 & $18(15.4 \%)$ & $103(22.3 \%)$ & 0.1006 & $6(30.0 \%)$ & $37(26.2 \%)$ & 0.7221 \\
\hline $100 \mu \mathrm{g}$ & $78(56.9 \%)$ & $322(53.4 \%)$ & 0.4536 & $69(59.0 \%)$ & $244(52.8 \%)$ & 0.2323 & $9(45.0 \%)$ & $78(55.3 \%)$ & 0.3861 \\
\hline $200 \mu \mathrm{g}$ & $25(18.2 \%)$ & $74(12.3 \%)$ & 0.0636 & $25(21.4 \%)$ & $70(15.2 \%)$ & 0.10496 & 0 & $4(2.8 \%)$ & 0.9961 \\
\hline $\begin{array}{l}\text { Mean maximum } \\
\text { used dose }(\mu \mathrm{g})\end{array}$ & $104 \pm 52$ & $91 \pm 50$ & 0.0121 & $110 \pm 52$ & $96 \pm 52$ & 0.0052 & $65 \pm 34$ & $77 \pm 38$ & 0.1587 \\
\hline
\end{tabular}

RCA: right coronary artery, LCA: left coronary artery

structive coronary arteries was markedly higher than that in male patients $(110 \pm 52 \mu \mathrm{g}$ vs. $96 \pm 52 \mu \mathrm{g}, \mathrm{p}=0.0052)$. Furthermore, in patients with obstructive coronary arteries, there was no differences regarding the incidence of the maximum ACh dose used, and the mean maximum used ACh dose for provoked spasm between female and male patients. The mean maximum used dose of ACh except for female with RCA was markedly higher in patients without obstructive coronary arteries than in those with them. As shown in Sup- plementary material 1 , there was no differences regarding the mean maximum used dose of ACh on both coronary arteries in patients with and without a history of smoking or with and without obstructive coronary arteries between male and female patients.

\section{Comparisons of major complications during positive ACh testing}

Major complications were observed in 37 patients (4.0\%) 


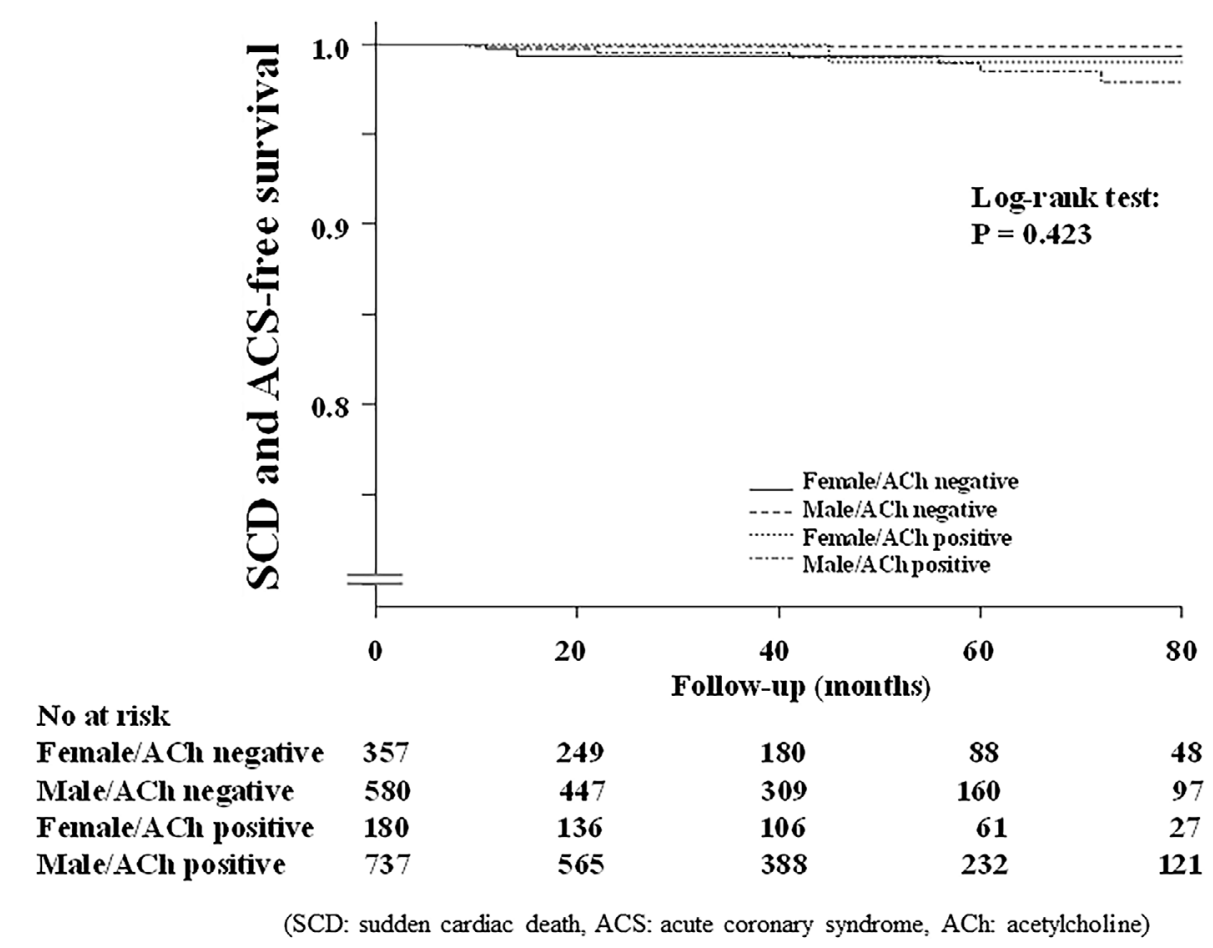

Figure 2. Kaplan-Meier survival curves for SCD and ACS in patients with and without positive spasm by ACh. SCD: sudden cardiac death, ACS: acute coronary syndrome, ACh: acetylcholine

including 11 female patients $(6.1 \%)$ and 26 male patients (3.5\%), as shown in Supplementary material 2. However, no difference was recognized between the sexes. Electrical defibrillation was necessary in five patients including one female patient $(0.6 \%)$ and four male patients $(0.5 \%)$, but the difference was not significant. Irreversible complications were not observed in either all 917 patients with provoked positive spasm or in the 937 patients with negative $\mathrm{ACh}$ tests.

\section{Comparisons of medications at baseline and clinical outcomes}

As shown in Table 2, in patients with ACh positive, the frequency of $\mathrm{CCB}$ was not different between the sexes, while the incidence of benzodiazepine type of CCB in male patients was significantly higher than that in female patients ( $26.7 \%$ vs. $17.2 \%, \mathrm{p}<0.01)$. The frequency of aspirin and nitrates in male patients was markedly higher than that in female patients, whereas there were no differences regarding the administration of angiotensin receptor blocker or angiotensin-converting-enzyme inhibitor, beta-blocker, antiplatelet, nitrate tape, nicorandil, or statin between the two groups. In contrast, in patients with $\mathrm{ACh}$ negative, the frequency of $\mathrm{CCB} /$ aspirin/nitrates were markedly higher in males than females. The clinical outcomes regarding the SCD and ACS free survival on both sexes under medications were not different as shown in Fig. 2. However, the MACEs free survival was markedly lower in male patients with ACh positive than in female patients with ACh negative as shown in Fig. 3.

\section{Discussion}

We herein reported the sex-related differences in Japanese patients with provoked positive spasm by ACh spasm provocation tests. Compared with male patients who had positive provoked spasm by ACh testing, female patients tended to be older, have less history of smoking, a higher left ventricular ejection fraction, a higher level of serum lipid data except for triglyceride, less provoked spasm in the left circumflex artery, more diffuse and combined type spasm. Furthermore, females had less ST elevation and more ST depression during ACh testing, while the mean used maximum ACh dose for positive provoked spasm in females was remarkably higher than that in males on both coronary arteries. Male may be more sensitive to ACh compared with female in Japanese populations.

We reported the clinical characteristics of female patients with coronary spastic angina (9). Compared with male patients, female patients with coronary spastic angina had less organic stenosis, less history of smoking, and fewer focal spasms. Kawano et al. also reported the gender differences in the clinical characteristics and outcomes of patients with vasospastic angina (10). As compared with male patients, female patients were characterized by an older age, a lower incidence of smoking, and less significant organic stenosis. Overall, the incidence of major cardiac events was not different between the two groups. Compared with previous studies, we found the same results except for organic stenosis in this study. However, in this study, we clarified the clinical characteristics of provoked positive spasm by ACh 


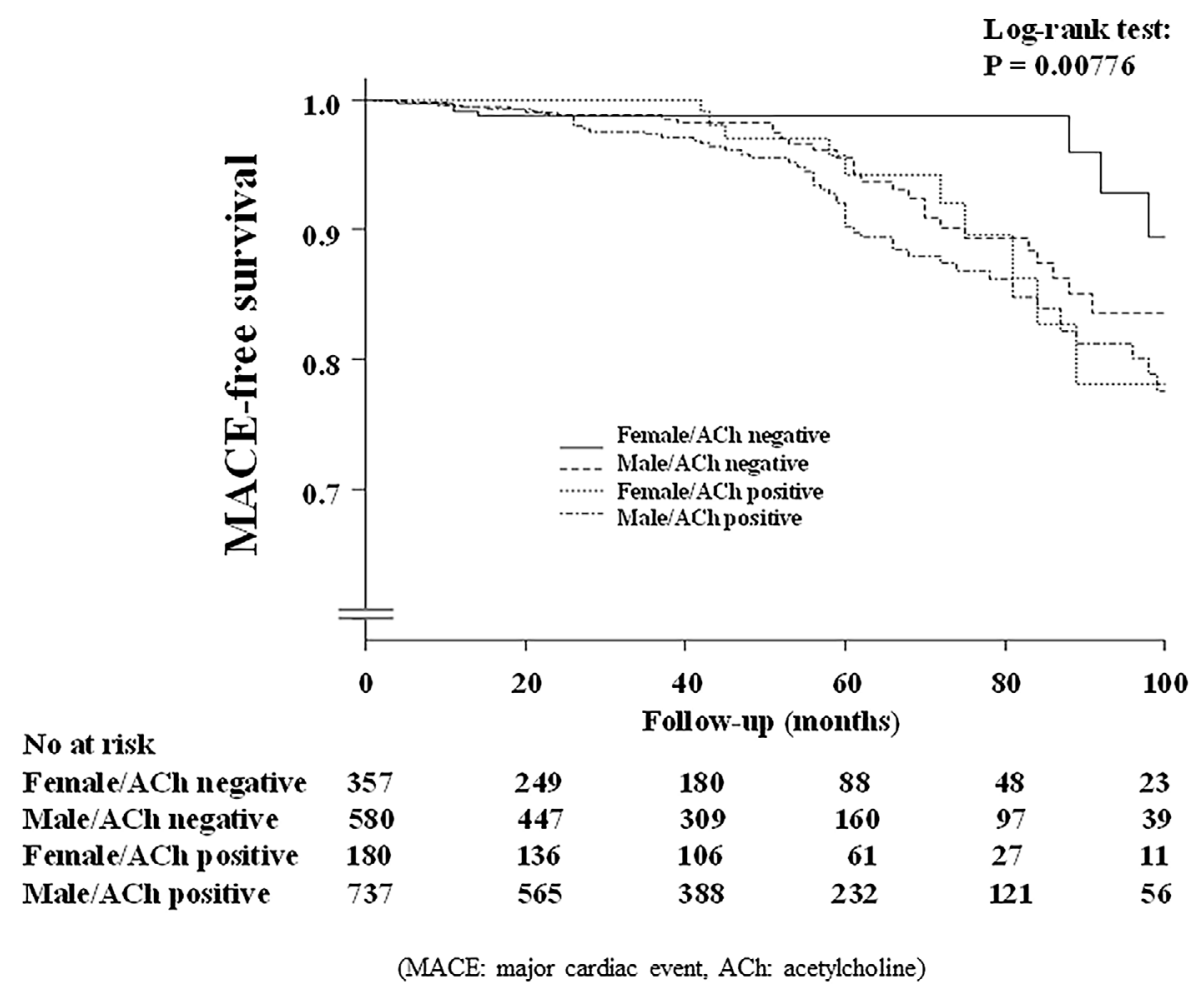

Figure 3. Kaplan-Meier survival curves for MACE in patients with and without positive spasm by ACh. MACE: major cardiac events, ACh: acetylcholine

but not by definite vasospastic angina.

Sato et al. reported that females had more diffuse spasm by ACh tests compared with males [56.5\% (218/386) vs. $29.6 \%$ (144/487), $\mathrm{p}<0.001]$ (11). In contrast, males had more focal spasm than females $[70.4 \%$ (343/487) vs. $43.5 \%$ (168/386), p <0.001]. Furthermore, the incidence of ST elevation during $\mathrm{ACh}$ tests in focal spasm group was significantly higher than that in diffuse spasm group ( $43 \%$ vs. $17 \%, \mathrm{p}<0.001)$, whereas the frequency of ST depression during ACh tests in the diffuse spasm group was markedly higher than that in the focal spasm group (83\% vs. 58\%, p< 0.001). We found results that were similar to those of Sato et al. in this study. However, Sato et al. did not mention the mean maximum $\mathrm{ACh}$ dose for provoked positive spasm in their study.

Aziz et al. reported the sex-related differences in the vasomotor function in European patients with angina and unobstructed coronary arteries (5). They concluded that European female patients have a higher prevalence of vasomotor dysfunction compared with male patients and a pathological ACh test was observed at lower ACh doses in females compared with males. However, in their study, a pathological ACh test was defined as epicardial spasm or coronary microvascular dysfunction. Furthermore, the incidence of coronary microvascular dysfunction in females was twice as high as that in males $(42.4 \%$ vs. $20.2 \%, \mathrm{p}<0.001)$ and the frequency of epicardial spasm in females was also significantly higher than that in males $(27.8 \%$ vs. $22.9 \%$, p< 0.05). In contrast, in our study, the incidence of provoked positive spasm by ACh tests in males is markedly higher than that in females $(56.3 \%$ vs. $32.6 \%, \mathrm{p}<0.001)$. In the European study, females were observed in approximately $60 \%$ of study cohort, while males were recognized in over $70 \%$ of our study subjects. There may be some selection bias of ACh spasm provocation tests between the two studies. Moreover, hypertension was found in over $70 \%$ of European study populations, whereas approximately $45 \%$ of study patients had hypertension in our Japanese peoples. According to the report of Sato et al, the frequency of provoked positive spasm by ACh tests in male patients was also significantly higher than that in female patients $[53.9 \%$ (487/903) vs. $45.0 \%$ (386/857), p <0.001] (10). This may be the most striking difference between Japanese and European populations by ACh testing. In our Japanese patients with ACh testing, the mean maximum ACh dose used for provoked positive spasm in females was markedly higher than that in males. However, the precise data of used ACh dose in just epicardial spasm patients was not shown in European patients. Japanese males may be more sensitive to ACh regarding the provoked positive spasm than Japanese females. Sex-related differences to ACh may be observed between European and Japanese populations. Further study is needed to clarify the sex-related differences of ACh spasm provocation tests among various species in the world.

\section{Limitations}

This study is associated with several limitations. First is the single center, retrospective, and small study samples. Second, there might be some selection biases regarding the indication of coronary angiography and ACh spasm provoca- 
tion test although we investigated consecutive populations. Third is that the study subjects were not all definite vasospastic angina or unobstructed coronary artery disease. We analyzed the clinical and angiographical findings, and clinical outcomes in patients with positive provoked spasm by ACh testing. Furthermore, there was no difference regarding the ischemic ECG changes and angiographical characteristics during ACh testing and the frequency of maximum ACh dose used for positive provoked spasm between all ACh positive patients and those with nonobstructive coronary arteries. However, the mean maximum used ACh dose for provoked positive spasm in the RCA was not different between male and female patients with unobstructed coronary arteries. Fourth is the methodological difference between Japanese and European patients. We performed ACh spasm provocation tests with a pacemaker according to the Japanese Circulation Society guidelines (6). However, European cardiologists administered intracoronary ACh for 3 minutes without pacemaker. They performed intracoronary ACh testing on both coronary arteries in just a third study subjects $(34 \%)$ of their previous study report (12).

\section{Conclusion}

The findings of our study suggest that males were more sensitive to ACh compared with females in Japanese populations, because ST elevation during ACh testing and focal provoked spasm was frequently observed in males than females, and the mean maximum used ACh dose for provoked positive spasm in males was significantly lower than that in females. A racial difference in the coronary response to ACh may be recognized between European and Japanese populations. In the future, cardiologists should try to further elucidate the sex differences in the coronary responses to ACh among various species based on cardiac catheterization studies.

The authors state that they have no Conflict of Interest (COI).

\section{References}

1. Yasue H, Horio Y, Nakamura N, et al. Induction of coronary artery spasm by acetylcholine in patients with variant angina : possible role of the parasympathetic nervous system in the pathogenesis of coronary artery spasm. Circulation 74: 955-963, 1986.

2. Okumura K, Yasue H, Matsuyama K, et al. Sensitivity and specificity of intracoronary injection of acetylcholine for the induction of coronary artery spasm. J Am Coll Cardiol 12: 883-888, 1988.

3. Okumura K, Yasue H, Horio Y, et al. Multivessel coronary spasm in patients with variant angina: a study with intracoronary injection of acetylcholine. Circulation 77: 535-542, 1988.

4. Sueda S, Miyoshi T, Sasaki Y, Sakaue T, Habara H, Kohno H. Gender differences in sensitivity of acetylcholine and ergonovine to coronary spasm provocation test. Herat Vessels 31: 322-329, 2016.

5. Aziz A, Hansen HS, Sechtem U, Prescott E, Ong P. Sex-related differences in vasomotor function in patients with angina and unobstructed coronary arteries. J Am Coll Cardiol 70: 2349-2358, 2017.

6. JCS joint working group. Guidelines for diagnosis and treatment of patients with vasospastic angina (coronary spastic angina) (JCS 2013). Circ J 78: 2779-2801, 2014.

7. Sueda S, Ochi N, Kawada H, et al. Frequency of provoked coronary vasospasm in patients undergoing coronary arteriography with spasm provocation test of acetylcholine. Am J Cardiol 83: 1186-1190, 1999.

8. Scanlon PJ, Faxon DP, Audet AM, et al. ACC/AHA guidelines for coronary angiography. A report of the American College of Cardiology/American Heart Association Task Force on Practice Guidelines (Committee on Coronary Angiography). J Am Coll Cardiol 33: 1756-1824, 1999.

9. Sueda S, Suzuki J, Watanabe K, et al. Clinical characteristics of female patients with coronary spastic angina: comparison with male patients. Jpn Circ J 64: 416-420, 2000.

10. Kawana A, Takahashi J, Takagi Y, et al. Gender differences in the clinical characteristics and outcomes of patients with vasospastic angina. Circ J 77: 1267-1274, 2013.

11. Sato K, Kaikita K, Nakayama N, et al. Coronary vasomotor response to intracoronary acetylcholine injection, clinical features, and long-term prognosis in 873 consecutive patients with coronary spasm: analysis of a single-center study over 20 years. J Am Heart Assoc 2: e000227, 2013.

12. Ong $P$, Athanasiadis A, Borgulya G, et al. Clinical usefulness, angiographic characteristics, and safety evaluation of intracoronary acetylcholine provocation testing among 921 consecutive white patients with unobstructed coronary arteries. Circulation 129: 17231730, 2014.

The Internal Medicine is an Open Access journal distributed under the Creative Commons Attribution-NonCommercial-NoDerivatives 4.0 International License. To view the details of this license, please visit (https://creativecommons.org/licenses/ by-nc-nd/4.0/).

(C) 2021 The Japanese Society of Internal Medicine

Intern Med 60: 2357-2365, 2021 